\title{
CRITERIOS REFERENCIALES Y SECTORIALES EN LA TOMA DE DECISIONES RELATIVAS AL TRATO DE LA INFANCIA Y LA ADOLESCENCIA EN EL ÁMBITO PÚBLICO LOCAL
}

\author{
MARINA ALARCÓN ESPINOZA* \\ LUCIO REHBEIN FELMER**
}

\begin{abstract}
RESUMEN
La presente investigación tuvo por objetivo caracterizar la toma de decisiones de quienes tienen la posibilidad de incidir en la condición de las personas menores de 18 años, en la comuna de Temuco. Lo anterior, considerando tanto los criterios a la base de la decisión como la perspectiva de futuro deseado para la infancia y la adolescencia. El estudio fue de carácter descriptivo, en un marco de metodología cualitativo, en el que se utilizó un diseño de casos múltiples en tres etapas: i) Encuesta, ii) Grupo Delphi, y iii) Taller Diagnóstico-Propositivo. En cada etapa se recopiló por separado la información de los sectores educación, salud, justicia y gobierno. Los resultados, muestran cuatro criterios operativos en la toma de decisiones, y dos prospectivos, asociados a la visión de futuro que los agentes responsables consideran en su proceso decisional. Estos resultados se discuten teniendo en consideración las dinámicas culturales e institucionales presentes, y la necesidad de una participación activa por parte de niños, niñas y adolescentes, en el desarrollo de sus localidades y regiones.
\end{abstract}

\section{Palabras ClaVe: Políticas PÚBliCas, DeRechos del niÑo, DESCENTRALIZACIÓN}

* Psicóloga, terapeuta familiar y Magíster en Desarrollo Humano a escala Local y Regional. Docente del Departamento de Psicología Universidad de La Frontera y psicóloga del Centro Comunitario de Salud Mental Familiar «La Rueda», Municipalidad de Temuco.

Correo electrónico: calarcon@ufro.cl.

** Psicólogo, Doctor en Psicología, docente Departamento de Psicología, Universidad de la Frontera, Temuco, Chile. 


\section{RESUMO}

\section{CRITÉRIOS REFERENCIAIS E SETORIAIS NA TOMADA DE DECISÕES RELATIVAS AO TRATAMENTO DA INFÂNCIA E DA ADOLESCÊNCIA NO ÂMBITO PUBLICO LOCAL}

A presente pesquisa teve por objetivo caracterizar a tomada de decisões daqueles que têm a possibilidade de incidir na condição das pessoas menores de 18 anos na comunidade de Temuco, considerando tanto os critérios na base da decisão como a perspectiva de futuro desejado para a infância e a adolescência. $\mathrm{O}$ estudo foi de caráter descritivo, em um marco de metodologia qualitativa, na qual se utilizou um desenho de casos múltiplos em três etapas: i) questionário, ii) grupo Delphi, e iii) oficina diagnóstico-propositiva. Em cada etapa recolheu-se separadamente informações dos setores de educação, saúde, justiça e governo. Os resultados mostram quatro critérios operativos na tomada de decisões e dois prospectivos, associados à visão de futuro que os agentes responsáveis consideram em seu processo decisório. Estes resultados são discutidos levando-se em consideração as dinâmicas culturais e institucionais presentes e a necessidade de uma participação ativa por parte de meninos, meninas e adolescentes no desenvolvimento de suas localidades e regiões.

PalaVRas ChaVe: Políticas PÚBliCAS, Direitos da CRIANÇA, DESCENTRALIZAÇÃO

\section{AbSTRACT}

\section{REFERENTIAL AND SECTORIAL CRITERIA ON RELATIVE DECISION MAKING IN DEALING WITH CHILDHOOD AND ADOLESENCE IN THE LOCAL PUBLIC AREA}

The present investigation had by objective to characterize decision making of those who have the possibility of bearing on people under the age of 18 years, in the commune of Temuco. Previously, as much as considering the criteria to the base of the decision as the perspective of future wished for childhood and adolescence. The study was of descriptive character, in a qualitative frame of methodology, in which a design of multiple cases in three stages was used: i) survey, ii) Delphi group, and iii) diagnosis-propositivo workshop. In each stage the information of the sectors was compiled separately, education, health, justice and government. The results, show four operative criteria in decision making, and two prospective, associated to the future vision that the responsible agents consider in their decisional process. These results are discussed, taking in consideration the present cultural and institutional dynamics, and the necessity of an active participation on the part of children and adolescents, in the development of their localities and regions.

KEY WORDS: PUBliC POLITICS, CHILDREN's RIGHTS, DECENTRALIZATION 


\section{INTRODUCCIÓN}

EL DESARROLLO HUMANO HA tenido un fuerte énfasis en la última parte del siglo XX, lo que se ha referido específicamente a la necesidad de colocar al centro del mismo al individuo (persona), observándose entre sus implicancias, la necesidad de cautelar el respeto por los derechos humanos y el fortalecimiento de la democracia y de los gobiernos locales, el aumento de la participación de la sociedad civil y la constitución de la ciudadanía. Lo cual implica revisar el modo de hacer sociedad y el papel que cada integrante, desempeña en ésta.

Paralelamente, en este mismo período surgió también una mayor preocupación por los niños, niñas y adolescentes, la cual se ha traducido recientemente en la generación de un instrumento de aplicación de los derechos humanos a este segmento etario, denominado Convención Internacional por los Derechos del Niño (Unicef, 2001).

La Convención es portadora de la idea de un nuevo trato hacia la infancia y la adolescencia, toda vez que considera personas titulares de derechos, a los niños, niñas y adolescentes, lo que tiene implicancias respecto a las relaciones que se establecen con el mundo adulto y con respecto de su participación en el quehacer social.

La implementación de la Convención ha tenido avances y retrocesos, observándose que se trata de un proceso en el que aún queda mucho por avanzar. En este contexto, una de las necesidades por atender se refiere a que las personas que toman decisiones que inciden en la aplicación de políticas públicas dirigidas a los niños, niñas y adolescentes, se comprometan efectivamente con los principios y el espíritu de la Convención (Unicef, 2002).

En Chile, durante la década de los 90 se trabajó en la reorientación de políticas públicas, en particular de las principales políticas sociales relacionadas con la situación de la infancia y la adolescencia, de manera de contribuir con ello a abordar de manera más integral la calidad de vida de los niños, niñas y jóvenes. Asimismo, más recientemente, se han diseñado, orientado y gestionado iniciativas innovadoras, favoreciendo la coordinación de las políticas sociales dirigidas a grupos prioritarios, entre los que se encuentra la infancia y la adolescencia (Gobierno de Chile, 2001).

Sin embargo, cabe señalar que de acuerdo a los diversos estudios y análisis efectuados, aunque los avances son considerables, todavía existen problemas que no están siendo tratados de manera consecuente con la Convención. Esto se debe principalmente a tres factores i) a 
que, por una parte, el diseño y operacionalización de los programas sociales no siempre responde a una concepción basada en los derechos de los niños; ii) a que aún persisten algunos vacíos, inadecuaciones y contradicciones en las normativas legales vigentes; y iii) a que quienes han de tomar las decisiones necesarias para diseñar y/o ejecutar las acciones innovadoras enfrentan también los desafíos propios de un cambio de paradigma (Ramírez, 2002).

De especial interés en el marco de la Convención por los derechos del niño son los derechos civiles y políticos. Si bien la Convención habla de la necesidad de atender en forma integral todos los derechos, aquellos derechos más vinculados a la provisión y la protección, han sido tradicionalmente objeto de las políticas públicas a través de variados dispositivos; mientras que, en el ámbito de los derechos civiles y políticos es donde se observa la necesidad de mayores innovaciones, pues es allí donde se pone en juego hasta qué punto estamos dispuestos, como sociedad, a favorecer la participación activa de niños, niñas y adolescentes.

Concordante con lo anterior, y a modo de ejemplo, cabe destacar que «una política de juventud es aquella que tiene como horizonte posible a sujetos integrales y propositivos y que, desde ese accionar se atiendan necesidades y dimensiones de sus vidas personales y colectivas». Esto implica, fundamentalmente que una política de juventud, debe tener un enfoque integral, positivo, propositivo y local (Silva, 2000).

Integral, en el sentido que debe dar cuenta a las variadas interrogantes y necesidades que las y los jóvenes expresan, sean éstas de orden material, simbólica, culturales, de pertenencia, de proyectos de vida, de trabajo, de afecto y de relaciones, entendiéndolas como puntos de partida en el proceso de construcción del vínculo entre los jóvenes y la institucionalidad pública, elemento relevante en el proceso de construcción ciudadana (Silva, 2000).

Positiva, en tanto debe asumir una óptica centrada en los aspectos positivos de las y los jóvenes de la comuna, lo que implica el desarrollo de un discurso institucional coherente con esta óptica.

Propositiva, en cuanto capaz de reconocer en las diversas propuestas de acciones, organizativas, y de interés, los elementos potenciadores que ellas tienen, como expresión de sus propias formas y modelos de intervención en sus espacios locales, y por sobre todo de las capacidades que poseen los propios jóvenes en sus diversos espacios y realidades (Silva, 2000). 
Locales, en la medida que en los espacios local/territorial puede concretizarse y visualizarse de manera cotidiana y cercana una política de juventud de carácter integral e integrada (no sectorial), por la cercanía entre sujetos juveniles e instancias públicas. Es el lugar donde el sujeto juvenil puede hacerse real y ejercer su calidad de ciudadano. Es el espacio, el lugar donde ejercer la ciudadanía a «escala humana» (Silva 2000).

Al respecto Allan (1999), plantea que el empoderamiento infanto-juvenil debe entenderse como el pasar el poder y la responsabilidad de las decisiones a esta población, en la medida de sus capacidades. Además, debe basarse en la formación de líderes bien informados y capaces de analizar y resolver los problemas de la comunidad que afectan sus necesidades como niños y jóvenes. Para lograr lo anterior, sin embargo, resulta necesario contar con la voluntad política y el compromiso social, sobre todo de las personas claves a nivel local y comunal.

Considerando los antecedentes antes expuestos, el presente estudio se pregunta $¿$ En qué basan sus decisiones y cómo proyectan los futuros escenarios de la infancia y adolescencia en la comuna de Temuco, quiénes tienen la posibilidad de incidir en la implementación de la Convención Internacional por los Derechos del Niño?

Más específicamente, la presente investigación busca contribuir a dilucidar los criterios que se encuentran a la base de la toma de decisiones referentes a los menores de 18 años de la comuna de Temuco, así como la visión de futuro deseado desde la cual se implementan tales decisiones. Se espera que los hallazgos del estudio contribuyan al diseño y evaluación de estrategias más efectivas y eficientes que promuevan el desarrollo infanto-juvenil, con una mirada en el presente, pero sobre todo con una perspectiva de largo plazo.

\section{MÉTODO}

Participantes. La población en referencia para la investigación corresponde a los funcionarios de instituciones públicas y privadas que trabajan y/o toman decisiones vinculadas a temas de infancia y adolescencia en Temuco. Mediante un muestreo intencionado con base en criterios de relevancia política y oportunidad con autoselección y confirmación posterior, se eligieron las muestras de decisores, que finalmente participaron en cada una de las etapas del estudio. 
Procedimiento. El trabajo de investigación contempló tres fases diferentes en la obtención de resultados: i) la encuesta preliminar; ii) la realización del grupo Delphi; y iii) la realización del taller diagnóstico-propositivo. Estas etapas se desarrollaron secuencialmente, obteniéndose en las dos primeras los insumos necesarios para la realización de las etapas siguientes (ii y iii, respectivamente). En la primera fase se convocaron 50 participantes y respondieron 35; en la segunda fase, se convocaron 40 participantes y respondieron 16; y en la tercera y última fase, se convocó a los mismos 40 participantes de la segunda fase, y respondieron 26.

En cada etapa se invitó a los participantes a través de una convocatoria escrita avalada por la Universidad, la Secretaría Regional de Planificación y Coordinación (Serplac IX Región) y por Unicef.

Recolección de datos. Como se ha señalado, los datos fueron recogidos en tres etapas, utilizando en cada una de ellas distintas estrategias de recolección y formas de registro de los datos. En una primera etapa se envió una encuesta a los directores y/o encargados de instituciones o programas que trabajan con niños y jóvenes en la ciudad de Temuco. En la encuesta se consultó respecto de la Convención Internacional por los Derechos del Niño y su implementación en la comuna, con las siguientes preguntas: ¿Qué conoce usted de la Convención Internacional por los Derechos del Niño?¿De qué modo cree usted que ha influido la citada Convención en el modo como su institución o programa aborda la labor desarrollada?¿Cree usted que sea necesario considerar, dentro de los derechos planteados en la Convención, la existencia de derechos civiles y políticos? ¿Por qué?

Adicionalmente se solicitó un listado de personas consideradas como decisores respecto del tema, en Temuco.

La segunda etapa consistió en la realización de un grupo Delphi. ${ }^{1}$ En términos específicos, se les envío a los decisores mencionados por los informantes de la primera etapa, vía correo electrónico o correo tradicional, i) la información sistematizada de esa etapa con el propósito de solicitar su opinión respecto de la Convención por los Derechos del Niño; ii) pedirles un comentario respecto de los datos preliminares recopilados en dicha etapa; y iii) una caracterización del mo-

1 La técnica Delphi consiste en la aplicación de cuestionarios sucesivos a expertos a fin de poner de manifiesto sus eventuales convergencias de opinión, permitiendo el surgimiento de consensos en torno a temas específicos (Godet, 1996). 
do en que ha enfrentado o proyecta enfrentar su toma de decisiones en el cargo que le corresponde ejercer.

La información recopilada sistematizada en un solo documento se reenvió, a los participantes a fin de que estos corroboraran lo señalado y/o entregaran nuevos énfasis con relación a la información señalada en su primer grupo de respuestas. Los participantes respondieron utilizando las mismas vías.

En la tercera etapa, una vez analizada la información del grupo Delphi, se efectuó un taller diagnóstico-propositivo, al cual se invitó a los participantes del Delphi, se les expuso los resultados de éste, y se les dividió según el sector de la administración pública en que trabajan - gobierno, justicia, educación y salud-. A los cuatro grupos resultantes se les presentaron distintas problemáticas (asociadas a su sector), en las que debían tomar decisiones respecto de niños, niñas y adolescentes. Al mismo tiempo se les consultó respecto de la consideración de la opinión de niños, niñas y adolescentes en la resolución de los problemas en cuestión.

Finalmente y luego de exponer respecto de las tendencias de la situación de la infancia en la región y la comuna, se les preguntó respecto del futuro deseado para niños y adolescentes en los próximos diez años y los responsables de las decisiones que en ultima instancia debiesen implementar este futuro deseado.

Los datos utilizados en cada una de las etapas recién descritas fueron datos textuales, entregados en forma escrita o registrados en cintas de audio, las que fueron luego transcritas para su análisis.

Análisis de los resultados. Los resultados de la primera etapa fueron analizados mediante un análisis de contenidos, junto con lo cual se realizó un listado con los decisores señalados, constituyéndose, de este modo, la muestra para la siguiente etapa.

Con relación a los resultados del Delphi, se efectúo un análisis de contenidos a los resultados de la primera vuelta, que permitió la sistematización de los discursos y la realización de una síntesis integrada que se reenvió a los participantes, solicitando reacciones por parte de los éstos. Los datos recogidos en la segunda vuelta, fueron nuevamente analizados mediante análisis de discurso.

Finalmente los datos recogidos en el Taller y transcritos posteriormente fueron analizados mediante análisis de contenidos efectuando en primera instancia una codificación libre por parte de la investigadora y dos evaluadores externos. Dicha información fue categorizada permitiendo la elaboración de árboles de categorías, en base a las 
cuales se recategorizaron los datos y se aplicó el Método de Análisis Jerárquico Ponderado (Pérez-Luco, 2002).

Criterios de validación y resguardos éticos. En las tres etapas de la investigación los participantes dieron a conocer sus opiniones de manera voluntaria. Durante la primera y segunda etapa de esta investigación la participación de los encuestados fue anónima. En la segunda etapa los resultados preliminares fueron reenviados a los decisores de modo que ellos corroboraran que lo señalado era efectivo, mientras que durante el análisis de los resultados de la tercera etapa se trianguló la codificación de los datos.

\section{Resultados}

Fase 1: al tabular los datos de la encuesta administrada en la primera fase, se constató que, en términos formales, la mayor parte de los encuestados (24 de 35), reportó conocer «datos" en relación a la suscripción de Chile, a las fechas o instituciones asociadas a la Convención, al número de artículos o de derechos. Algunos de estos «datos», son erróneos. Una menor proporción de los encuestados (4 de 35), señaló que la Convención de los Derechos Humanos, planteando la necesidad de cambiar la forma de vivir en sociedad. Algunos de ellos hicieron alusión a los niños en términos de «sujeto de derechos» $\mathrm{y}$ "ciudadano».

Referente a la implementación de acciones propiciatorias del cumplimiento de la Convención, se observaron diferentes énfasis, dependiendo del sector de pertenencia de los encuestados. De este modo, el sector salud reportó en mayor medida labores de promoción, prevención y denuncia de casos, mientras que educación planteó, en algunos casos, que el tema estaba presente en los objetivos transversales y/o en el proyecto institucional, o bien que no existía una propuesta específica. Entre los participantes de este sector, se hizo mención reiterada a la necesidad de proteger a los niños de abusos y a su vez a la necesidad de enseñar y promover, en los niños y niñas, tanto derechos, como deberes. Educación parvularia, por su parte, planteó una labor más dedicada a la difusión entre los adultos de los derechos mencionados en la Convención y su preocupación por el mejoramiento de oportunidades para los niños y niñas.

En relación a la necesidad de considerar los derechos civiles y políticos de los niños, se mencionaron términos tales como «ser humano», «persona en sociedad», «sociedad civil», «persona», y «ciudadano». Junto con ello, se sugirió la posibilidad que al contar la infancia con este 
tipo de derechos, se pueda incidir políticamente, a través de leyes que beneficien a los niños. En menor medida, se consideró la relación entre este hecho y el fortalecimiento de los valores democráticos.

En cuanto a las personas percibidas como relevantes en el ámbito de la toma de decisiones comunales en relación a los derechos de niños, niñas y adolescentes, se mencionaron 32 cargos, o personas que ejercían dichos cargos; entre ellos, el alcalde y los concejeros municipales; los Seremis de justicia, salud y educación; los directores de servicios públicos como Sename, Integra, Junji, Junaeb, Injuv, Conace, Chile Deportes, Salud Araucanía Sur, Médico Legal y Gendarmería; los parlamentarios por la circunscripción y distrito; y los directores de Didecos, OPD, Cosam «La Rueda», Clínica Psicológica de la UFRO y CDT Belén-Alborada.

En la segunda etapa, los participantes del grupo Delphi, señalaron avances y retrocesos en la implementación de la Convención Internacional por los Derechos del Niño, acciones institucionales favorecedoras y pendientes, además de mencionar la necesidad de cambios estructurales en las instituciones. Junto con lo anterior, hicieron mención a que las acciones y decisiones que se requieren, muchas veces van más allá de su marco de acción, por lo cual otorgaron importancia a la forma en que se toman las decisiones y a la perspectiva desde la cual se aborda el tema de la infancia y adolescencia en la comuna. De este modo, en la tercera etapa se buscó, mediante la realización del taller abordar los criterios presentes en la toma de decisiones y las perspectivas del futuro deseado para niños, niñas y adolescentes de la comuna.

En la tercera etapa, se indagó respecto de los dos grandes ámbitos, antes mencionados, considerándose dos dimensiones en el proceso de toma de decisiones, la primera de ellas sería la operativa, referida a los criterios en la toma de decisiones respecto de niños, niñas y adolescentes, en la comuna. La segunda dimensión sería la prospectiva referida al futuro que estos mismos decisores desean para los(as) niños, niñas y adolescentes de la comuna.

En el aspecto operativo, emergen cuatro categorías: El criterio normativo entendido como, el conjunto de normas y valores que determinan una materia o actividad; el criterio experto que indica la valoración por la habilidad y/o experticia de profesionales o equipos, en una determinada técnica o materia; el criterio evolutivo, refiere la consideración por el desarrollo psicosocial de niños, niñas y adolescentes y, el criterio administrativo entendido como la valoración por el modo habitual de proceder en el abordaje institucional. 
En la muestra total de los asistentes al taller se observó que el criterio «normativo», sería el más utilizado por los decisores participantes, superando por más del doble a los otros tres criterios; siendo el segundo más utilizado el criterio «administrativo». Los criterios «experto»y «evolutivo», se utilizaron en una similar proporción (Tabla 1).

Tabla 1

Distribución de códigos operativos por categorías generales y subgrupos

\begin{tabular}{|l|c|c|c|c|c|c|c|c|}
\hline & \multicolumn{2}{|c|}{$\begin{array}{c}\text { Criterio } \\
\text { Normativo }\end{array}$} & \multicolumn{2}{c|}{$\begin{array}{c}\text { Criterio } \\
\text { Experto }\end{array}$} & \multicolumn{2}{c|}{$\begin{array}{c}\text { Criterio } \\
\text { Evolutivo }\end{array}$} & \multicolumn{2}{c|}{$\begin{array}{c}\text { Criterio } \\
\text { Administrativo }\end{array}$} \\
\hline & $\mathrm{n}$ & $\%$ & $\mathrm{n}$ & $\%$ & $\mathrm{n}$ & $\%$ & $\mathrm{n}$ & $\%$ \\
\hline TOTAL & 225 & 44.1 & 93 & 18.2 & 90 & 17.6 & 102 & 20.0 \\
\hline Salud & 88 & 71.0 & 19 & 15.3 & 9 & 7.3 & 8 & 6.5 \\
\hline Educación & 46 & 31.7 & 28 & 19.3 & 21 & 14.5 & 50 & 34.5 \\
\hline Justicia & 37 & 38.9 & 12 & 12.6 & 35 & 36.8 & 11 & 11.6 \\
\hline Gobierno & 54 & 37.0 & 34 & 23.3 & 25 & 17.1 & 33 & 22.6 \\
\hline
\end{tabular}

$\mathrm{n}$ : frecuencia de códigos asignados en la categoría por subgrupo.

Si bien, tal como señala la Tabla 1, todos los sectores resaltaron el criterio normativo, es necesario también destacar que en el sector Educación el criterio administrativo apareció priorizado, aunque ambos criterios muestran un escaso margen de diferencia. En el sector justicia, por su parte, se destaca la alta frecuencia con que, luego del criterio normativo, se hace mención al criterio evolutivo.

Profundizando en el análisis de los criterios presentes, en el ámbito operativo, de la toma de decisiones, se observa que del criterio normativo, emergen cuatro subcategorías:

i) La eficiencia, entendida como la necesidad de llegar a una real solución de la problemática; «es importante rescatar la cultura y costumbres del niño, porque si se toma una decisión arbitraria, ponle tú que el niño lo más probable es que él se escape o se vaya, ¿me entiendes?; o sea igual por el éxito de las decisiones que se tomen»; es la señalada con mayor énfasis en los sectores de educación, justicia y gobierno.

ii) La integración social que se entiende como la necesidad de ajustar a los miembros de la sociedad a lo señalado por la mayoría, «la única manera que nosotros veíamos, por lo menos en ese caso específico, es poder reorientar al niño y sacarlo del sector, sacarlo de donde 
está»; es la más referida por el sector salud y señalada con importancia en los sectores de educación y gobierno.

iii) La perspectiva de derechos que se refiere a la consideración del nuevo paradigma de derechos de las personas; «podría llegar a ser fundamental la opinión del niño, reconociendo sus lazos afectivos, creo yo. Podrían cambiar totalmente las decisiones que se están pensando desde afuera»; es planteada con relevancia en el sector salud y en alguna medida por justicia.

iv) La familia que se refiere a la perspectiva que releva el rol de la familia al momento de intervenir y requerir la solución de las problemáticas de niños, niñas y adolescentes «yo creo que podría ser una buena alternativa también el generar espacios de capacitación para las familias»; que se plantea en todos los sectores a excepción del sector justicia.

Tabla 2

Subcategorías del criterio normativo

\begin{tabular}{|l|c|c|c|c|c|c|c|c|}
\hline & \multicolumn{2}{|c|}{ Eficiencia } & \multicolumn{2}{c|}{$\begin{array}{c}\text { Perspectiva } \\
\text { de derechos }\end{array}$} & \multicolumn{2}{c|}{$\begin{array}{c}\text { Integración } \\
\text { social }\end{array}$} & \multicolumn{2}{c|}{ Familia } \\
\hline & $\mathrm{n}$ & $\%$ & $\mathrm{n}$ & $\%$ & $\mathrm{n}$ & $\%$ & $\mathrm{n}$ & $\%$ \\
\hline TOTAL & 74 & 32.9 & 47 & 20.9 & 65 & 28.9 & 39 & 17.3 \\
\hline Salud & 19 & 21.6 & 23 & 26.1 & 25 & 28.4 & 21 & 23.9 \\
\hline Educación & 15 & 32.6 & 8 & 17.4 & 14 & 30.4 & 9 & 19.6 \\
\hline Justicia & 18 & 48.6 & 10 & 27.0 & 9 & 24.3 & 0 & 0.0 \\
\hline Gobierno & 22 & 40.7 & 6 & 11.1 & 17 & 31.5 & 9 & 16.7 \\
\hline
\end{tabular}

n: frecuencia de códigos asignados en la categoría por subgrupo.

Por su parte del criterio experto emergieron las subcategorías calidad profesional en la que se enfatiza la calidad y experticia de los profesionales y técnicos en la búsqueda de soluciones «A veces también pasa porque tú no tienes las especialidades, por ejemplo nosotros no tenemos personas expertas en tratar estos temas (drogas)»; y los modelos de intervención que se refieren a la relevancia de las miradas teóricas y las metodologías utilizadas al momento de intervenir «más que lo de la capacitación a la familia, hablaría de un trabajo con la familia, que no necesariamente tenga que ver con capacitación... siempre estamos con la idea de que a la familia le faltan cosas».

En la muestra total, la mayor proporción de contenidos se refiere a la preocupación por los modelos de intervención, lo cual es mani- 
fiesto en casi todos los sectores, apreciándose una escasa relevancia de la subcategoría calidad profesional. La situación opuesta, la muestra el sector Justicia quien releva la validación de la calidad profesional, no mostrando contenido alguno en la subcategoría modelos de intervención.

Tabla 3

Subcategorías del criterio experto

\begin{tabular}{|l|c|c|c|c|}
\hline & \multicolumn{2}{|c|}{ Calidad Profesional } & \multicolumn{2}{c|}{ Modelos de Intervención } \\
\hline & $\mathrm{n}$ & $\%$ & $\mathrm{n}$ & $\%$ \\
\hline ToTAL & 17 & 18.3 & 76 & 81.7 \\
\hline Salud & 1 & 5.3 & 18 & 94.7 \\
\hline Educación & 3 & 10.7 & 25 & 89.3 \\
\hline Justicia & 12 & 100 & 0 & 0.0 \\
\hline Gobierno & 1 & 2.9 & 33 & 97.1 \\
\hline
\end{tabular}

n: frecuencia de códigos asignados en la categoría por subgrupo.

En el criterio evolutivo, emergen las subcategorías de: i) Consideración de estadios evolutivos, entendida como la valoración de las necesidades evolutivas de niños, niñas y adolescentes «justificar que la niña verbalizó... para mí que en ese tema, a los 11 años, es muy difícil que esté mintiendo»; es la más relevada por el sector salud y la más escasamente mencionada por el sector justicia. ii) Niño víctima referido a la percepción de que en la búsqueda de una solución, el niño termina siendo victima «uno de los aspectos que se evalúo en la última reunión de gabinete sectorial, a propósito de la reforma procesal penal, fue la doble o triple victimización de las personas (y en esto los niños) que son víctimas de abusos sexuales, porque primero le relatan a los carabineros, después al fiscal, después al médico legal»; es la mayormente mencionada por los sectores de educación y justicia, siendo expresada con menor frecuencia por el sector gobierno. iii) Baja credibilidad en los niños, referida como la escasa o nula validación del relato y/o las expresiones de los niños, niñas y adolescentes «Las pandillas se pueden diluir o transformar en algo más positivo, pero efectivamente no lo habíamos considerado, o sea, para nosotros no tienen, ni siquiera...no es que no tengan valor político, no tienen poder de decisión alguna, aparentemente los menores de 18 años, sobre su realidad»; sería la más señalada por el sector gobierno, no observándose contenidos de esta subcategoría en el sector salud. 
Tabla 4

Subcategorías del criterio evolutivo

\begin{tabular}{|l|c|c|c|c|c|c|}
\hline & \multicolumn{2}{|c|}{$\begin{array}{c}\text { Consideración de } \\
\text { estadios evolutivos }\end{array}$} & \multicolumn{2}{c|}{$\begin{array}{c}\text { Niño } \\
\text { Víctima }\end{array}$} & \multicolumn{2}{c|}{$\begin{array}{c}\text { Baja credibilidad } \\
\text { en los niños }\end{array}$} \\
\hline & $\mathrm{n}$ & $\%$ & $\mathrm{n}$ & $\%$ & $\mathrm{n}$ & $\%$ \\
\hline ToTAL & 19 & 21.1 & 43 & 47.8 & 28 & 31.1 \\
\hline Salud & 7 & 77.8 & 2 & 22.2 & 0 & 0.0 \\
\hline Educación & 3 & 14.3 & 14 & 66.7 & 4 & 19.0 \\
\hline Justicia & 2 & 5.7 & 23 & 65.7 & 10 & 28.6 \\
\hline Gobierno & 7 & 28.0 & 4 & 16.0 & 14 & 56.0 \\
\hline
\end{tabular}

$\mathrm{n}$ : frecuencia de códigos asignados en la categoría por subgrupo.

En relación al criterio administrativo, emergen las subcategorías de: i) Ajuste a los procedimientos institucionales, entendida como la necesidad de que la solución se ajuste a los procedimientos y/o reglamentos establecidos "Ahora yo no sé si se puede echar indirectamente a las niñas, sugerirles a los papás que cambien a las niñitas de colegio donde se sientan más cómodas... Si se puede, si está en el reglamento interno y estrictamente especificado porque caso tendría que retirarse a la niña»; es ampliamente señalada en los sectores de justicia, salud y educación, mostrándose en el sector gobierno en una segunda prioridad. ii) Delimitación de responsabilidad, señalada como la necesidad de delimitar a quien le corresponde la solución del problema para derivarlo «Los profesores solos, no van a poder, por ejemplo trabajar problemas conductuales porque no les compete. Lo mismo ocurre con el psicólogo, puede trabajar la parte conductual pero no va a poder trabajar la parte pedagógica, porque no le compete»; es señalada por el sector educación y en alguna medida por el sector gobierno, no observándose contenidos al respecto en los otros sectores. iii) Aprovechamiento de oportunidades entendido como el aprovechamiento de las oportunidades existentes en el medio «le puedes dar todas las oportunidades pero no las va a tomar»; es bastante poco señalado por todos los sectores, mostrándose algunos contenidos en los sectores de gobierno y educación. iv) Obstáculos del sistema, que se refiere a la visualización de los obstáculos, ante la implementación de las decisiones «Distinto es cuando uno tiene el problema ahí y como actúa con el problema ahí, porque uno puede explicar, puede entenderlo, puede analizarlo en documentos pero cuando tú tienes que actuar»; es planteado como primera prioridad en el sector gobierno, observándose en todos los sectores. 
Tabla 5

Subcategorías del criterio administrativo

\begin{tabular}{|l|c|c|c|c|c|c|c|c|}
\hline & \multicolumn{2}{|c|}{$\begin{array}{c}\text { Ajuste a los } \\
\text { procedimientos } \\
\text { institucionales }\end{array}$} & \multicolumn{2}{l|}{$\begin{array}{l}\text { Delimitación de } \\
\text { responsabilidad }\end{array}$} & \multicolumn{2}{l|}{$\begin{array}{l}\text { Aprovechamiento } \\
\text { de oportunidades }\end{array}$} & $\begin{array}{c}\text { Obstáculos } \\
\text { del sistema }\end{array}$ \\
\hline & $\mathrm{n}$ & $\%$ & $\mathrm{n}$ & $\%$ & $\mathrm{n}$ & $\%$ & $\mathrm{n}$ & $\%$ \\
\hline ToTAL & 47 & 46.1 & 21 & 20.6 & 4 & 3.9 & 30 & 29.4 \\
\hline Salud & 5 & 62.5 & 0 & 0.0 & 0 & 0.0 & 3 & 37.5 \\
\hline Educación & 25 & 50.0 & 17 & 34.0 & 1 & 2.0 & 7 & 14.0 \\
\hline Justicia & 9 & 81.8 & 0 & 0.0 & 0 & 0.0 & 2 & 18.2 \\
\hline Gobierno & 8 & 24.2 & 4 & 12.1 & 3 & 9.1 & 18 & 54.5 \\
\hline
\end{tabular}

n: frecuencia de códigos asignados en la categoría por subgrupo.

En el aspecto prospectivo, desde el cual se toman las decisiones, es decir, respecto del futuro que los decisores desean para la infancia y adolescencia de la comuna, se observan dos grandes categorías: la visibilización de los niños, niñas y adolescentes, que se refiere a la necesidad de verles, considerar sus necesidades y relevarlas al momento de tomar decisiones, y la existencia de mayores oportunidades para estos, entendida como el tener mayores y/o mejores posibilidades de desarrollo.

En términos totales, el énfasis planteado por los decisores está puesto en la búsqueda de mayores oportunidades. Esta situación se observa en prácticamente todos los sectores, manteniéndose similares proporciones, con la excepción del sector educación, el cual releva la visibilización de los niños.

Tabla 6

Distribución de códigos prospectivos por categorías generales y subgrupos

\begin{tabular}{|l|c|c|c|c|}
\hline & \multicolumn{2}{|c|}{$\begin{array}{c}\text { Visibilización } \\
\text { de los niños }\end{array}$} & \multicolumn{2}{c|}{$\begin{array}{c}\text { Mayores } \\
\text { oportunidades }\end{array}$} \\
\hline & $\mathrm{n}$ & $\%$ & $\mathrm{n}$ & $\%$ \\
\hline ToTAL & 250 & 43.8 & 321 & 56.2 \\
\hline Salud & 47 & 39.2 & 73 & 60.8 \\
\hline Educación & 110 & 59.8 & 74 & 40.2 \\
\hline Justicia & 44 & 40.7 & 64 & 59.3 \\
\hline Gobierno & 49 & 30.8 & 110 & 69.2 \\
\hline
\end{tabular}

n: frecuencia de códigos asignados en la categoría por subgrupo. 
Por su parte el sector gobierno, es el que con mayor énfasis señala la necesidad de mayores oportunidades, mostrando por tanto la frecuencia más baja en contenidos relativos a la visibilización de los niños, niñas y adolescentes.

Profundizando el análisis, en relación al futuro que los decisores desean para los niños, niñas y adolescentes de la región, se observa que en la categoría visibilización de los niños emergen las subcategorías cambio cultural, entendida como la promoción de un nuevo paradigma, más respetuoso de los derechos de las personas menores de 18 años «yo creo que lo que debiera ser es que de una vez por todas la sociedad se haga cargo de que está ejerciendo violencia y violación de derechos en los niños»; y las modificaciones al sistema político que se refiere a requerimientos a nivel político, necesarios para promover la visibilización de los niños «uno de mis sueños es que los municipios sean más autónomos e intencionen más en el diseño, no sólo en la ejecución e implementación».

Tabla 7

Subcategorías visibilización de los niños

\begin{tabular}{|l|c|c|c|c|}
\hline & \multicolumn{2}{|c|}{$\begin{array}{c}\text { Cambio } \\
\text { Cultural }\end{array}$} & \multicolumn{2}{c|}{$\begin{array}{c}\text { Modificaciones } \\
\text { al sistema político }\end{array}$} \\
\hline & $\mathrm{n}$ & $\%$ & $\mathrm{n}$ & $\%$ \\
\hline TOTAL & 156 & 62.4 & 94 & 37.6 \\
\hline Salud & 40 & 85.1 & 7 & 14.9 \\
\hline Educación & 78 & 70.9 & 32 & 29.1 \\
\hline Justicia & 16 & 36.4 & 28 & 63.6 \\
\hline Gobierno & 22 & 44.9 & 27 & 55.1 \\
\hline
\end{tabular}

n: frecuencia de códigos asignados en la categoría por subgrupo.

En la muestra total, la subcategoría cambio cultural, es la más señalada, siendo en los sectores de educación y salud, donde encuentra las más altas frecuencias, observándose una amplia diferencia con la subcategoría modificaciones al sistema político. Esta última, por su parte, se muestra priorizada por los sectores de justicia y gobierno.

En la categoría mayores oportunidades, emergen las subcategorías mejoramiento de la gestión publica que se refiere a la necesidad de mejorar el modo de implementar la política pública, optimizando los recursos «hay pocas redes de trabajo transparente», somos poco autocríticos, o sea «lo estamos haciendo fantástico», somos rígidos además 
porque claro, «nosotros lo hacemos bien pero las demás personas no ven su trabajo desde esta colina, no son lo suficientemente autocríticos», pero nosotros lo hacemos súper bien, "por eso te digo, ésas son las decisiones que hay que tomar, que las metas, que el diseño de la institución o de los programas vayan para ese lado porque ahora van para cualquier parte. Entonces tenemos una misión super bonita y las metas y los indicadores van para otro lado»y y el desarrollo humano, entendido como la necesidad de propiciar el desarrollo de las personas, tanto de los funcionarios que han de implementar las reformas, como de los niños y sus familias «pucha, o sea, con esa mentalidad es imposible que tú también logres la llegada con él y lo consideres como una persona y con alguien con sus derechos de opinión y todo... si está en un proceso de identidad. Además, históricamente siempre ha habido cabros con el pelo largo y todo el cuento... no sé qué pensar, igual hay un cuento de capacitación pero también de renovar, de cambio», «el sueño nuestro de hoy día ¿qué es? que en vez de tener el problema de alimentación que tenemos hoy día con esa gran cobertura, tengamos de programa Habilidades para la vida con una cobertura más alta».

Tabla 8

Subcategorias mayores oportunidades

\begin{tabular}{|l|c|c|c|c|}
\hline & \multicolumn{2}{|c|}{$\begin{array}{c}\text { Mejoramiento de la } \\
\text { gestión pública }\end{array}$} & \multicolumn{2}{c|}{$\begin{array}{c}\text { Desarrollo } \\
\text { humano }\end{array}$} \\
\hline & $\mathrm{n}$ & $\%$ & $\mathrm{n}$ & $\%$ \\
\hline TOTAL & 156 & 48.6 & 165 & 51.4 \\
\hline Salud & 36 & 47.4 & 37 & 48.7 \\
\hline Educación & 31 & 41.9 & 43 & 58.1 \\
\hline Justicia & 33 & 51.6 & 31 & 48.4 \\
\hline Gobierno & 56 & 50.9 & 54 & 49.1 \\
\hline
\end{tabular}

n: frecuencia de códigos asignados en la categoría por subgrupo.

Si bien el margen de diferencia entre ambas subcategorías es bajo, se observa una mayor preocupación por el desarrollo humano tanto en la muestra total como en los sectores de educación y salud; mientras que la necesidad de un mejoramiento de la gestión pública es en mayor medida percibida por los sectores justicia y gobierno. 


\section{DISCUSIÓN Y CONCLUSIONES}

Los principales resultados del estudio lo constituyen la emergencia de cuatro categorías de criterios para la toma de decisiones en la dimensión operativa del proceso decisional y dos categorías en la dimensión prospectiva. El segundo hallazgo lo constituye las claras distinciones de énfasis entre los sectores educación, salud, justicia y gobierno, lo que sin duda, se relaciona con la existencia de lógicas distintas presentes en cada sector. Estas lógicas no pueden obviarse al momento de destinar esfuerzos para la coordinación inter-institucional en la búsqueda de un desarrollo integral de la infancia y adolescencia.

Al unificar aquellos criterios de decisión que tienen mayor relación con lo institucional (normativo y administrativo) y aquellos que tienen mayor vinculación con las personas (experto y evolutivo), se observa que la mayor prioridad dada por los decisores está centrada en su rol como funcionario institucional, mas allá de la evaluación a partir de las competencias técnicas dadas por la profesión o la consideración por las características de los niños, niñas y adolescentes afectados por la decisión. Esto se vincula con las rigideces mostradas por el aparato público, según Ramírez (2002) y Sánchez (2002), en relación al carácter normativo de la institucionalidad.

En este aspecto es llamativa la relevancia que el sector salud otorga a los criterios normativo y administrativo, lo cual pudiere estar vinculado a una estructura jerarquizada, en el cual están ampliamente diseñados los procedimientos a seguir y los conductos, por lo que, establecer relaciones democratizadoras pudiere ser algo más difícil de lograr.

Lo anterior, junto con ser parte del problema puede ser parte de la solución ya que se podría decir que los cambios a la normativa y procedimientos institucionales deberán tener un fuerte impacto en el cambio de prácticas e intervenciones que incorporen un mayor respeto de los derechos de las personas. Aunque, por lo mismo se puede considerar que las resistencias a tales cambios han de ser considerables.

Ello es relevante, pues siendo, según Luhmann (en Izuzquiza, 1990), los sistemas sociales autorreferentes, es preciso que las iniciativas innovadoras partan desde la lógica interna del sistema, por lo cual el punto pareciera ser el cómo introducir al sistema alguna información que le permita replantearse sus relaciones, complejizarse y adquirir las funciones necesarias para atender a una nueva lógica, que parece observarse de modo incipiente. En este aspecto pareciera relevante el hecho de que los mismos decisores se planteen la necesidad 
de considerar a los niños dentro de los grupos a los cuales esta dirigida la política pública; verles pudiera ser un primer paso en el camino de considerarles personas, sujetos de derechos y protagonistas en los procesos de desarrollo.

El sector justicia a su vez, releva el criterio evolutivo, apreciándose contenidos vinculados a la percepción del niño como víctima. Se estima que lo anterior pudiera estar relacionado con las discusiones que coyunturalmente se han presentando respecto a las modificaciones en la ley de delitos sexuales vinculadas a aumentar las sanciones (edad de la víctima) y/o la ley de responsabilidad penal juvenil.

De ser esto efectivo, el interés por los aspectos evolutivos aparecería a fin de satisfacer una necesidad del criterio normativo, como lo es la dictación de leyes, lo cual pudiera implicar una oportunidad, pues al cuestionarse esto el sistema necesariamente se complejiza e introduce nuevas informaciones. Esto, sin duda provocará nuevas inconsistencias, que se sumaran a las tantas existentes en el proceso modernizador del estado referidas a la lentitud en el desarrollo de nuevas funcionalidades, en un proceso de complejización.

En relación con los escenarios futuros respecto de los cuales se toman las decisiones, se observa que en su mayoría los sectores evalúan la necesidad de mayores oportunidades, lo que aparece ampliamente vinculado con la superación de la pobreza y el mejoramiento de las condiciones de vida. En este aspecto un elemento que pudiese visualizarse como novedoso es la preocupación por el desarrollo humano ya que esto se vincularía con la idea de la promoción del desarrollo no sólo a partir de la obtención de bienes sino también una apuesta al capital humano y al desarrollo de éste.

Sin embargo, las preocupaciones fundamentales siguen siendo del orden de los bienes y servicios, no observándose aún, como un elemento relevante la necesidad de que los actores sociales, incorporados los niños, niñas y adolescentes, sean activos promotores del desarrollo de sus comunidades y ejerzan su ciudadanía más allá del reclamo por un mal servicio o un mal producto.

Coherente con lo anterior es también relevante que aparezca menos mencionado, dentro del futuro deseado para la infancia y la adolescencia, la visibilización de éstos como sujetos con necesidades y por tanto como personas con derechos. En este aspecto se pudiera hipotetizar que lo más costoso del proceso es precisamente el cambio cultural que implica y que cuestiona el rol de los «adultos tomando decisiones por», que obedece en mayor medida a una lógica paternalista. 
En este aspecto es necesario cuestionarse hasta que punto los adultos han asumido su rol ciudadano y ejercen sus derechos, pues sin duda, la experiencia de ejercer la propia ciudadanía tiene una influencia directa dentro de las pautas de crianza de las futuras generaciones.

El único sector que prioriza la visibilización de los niños es educación, radicando su preocupación en el cambio cultural necesario. Esto se puede relacionar con el rol de transmisión cultural que se entrega a educación y/o con el hecho de que es el sector que mantiene una vinculación directa con los niños y adolescentes de la comuna. Sin embargo, resulta un tanto contradictorio que visualizando la necesidad de ver a los niños, niñas y adolescentes, al momento de realizar efectivamente el proceso de toma de decisiones prioricen por los criterios más vinculados a las necesidades institucionales.

En esto es importante destacar que en la primera etapa del estudio, este sector mostró, como estrategia para la implementación de la Convención, la presencia de sus principios en los objetivos transversales y/o en el proyecto educativo, es decir en las normativas institucionales, lo cual pudiese parecer concordante con el hecho de que en la tercera etapa este mismo sector releva los criterios administrativos y normativos en la toma de decisiones. Ello, si bien en un primer momento pudiere parecer coherente, se observa que no es garantía de la real implementación de los derechos de la infancia, puesto que así como puede estar presente dicha normativa, aparecen a su vez otras más arraigadas en la cultura institucional, tales como el perfil del alumno deseado, las pautas de disciplina, etc. Quedando, en muchas ocasiones, los derechos de los niños, niñas y adolescentes más en un nivel discursivo que en la cotidianeidad de la vida escolar.

Estas inconsistencias, en que aparece por una parte lo deseado y por otra la conducta decisional propiamente tal, se consideran, altamente desgastadoras para los profesionales involucrados y se podría relacionar con la percepción de obstáculos en la implementación de los cambios en los distintos sectores participantes del estudio. Dichos obstáculos, estarían entonces vinculados directamente con quienes deben implementar una nueva forma de considerar a la Infancia y la Adolescencia.

En este sentido, es viable plantearse la hipótesis de que estos sistemas autorreferentes, estarían mostrando una crisis y una necesidad creciente de cambios, que pudiesen estar relacionados con el futuro deseado, al mencionar dentro de sus sueños, tanto el mejoramiento de la gestión pública como las modificaciones al sistema político, ambos 
aspectos que serían de competencia del sistema político, que como tal agrupa a los sistemas o sectores mencionados. Esto se corresponde, a su vez con el futuro deseado por los decisores en relación a la necesidad de flexibilizar el modo de implementar las políticas coherentes favoreciendo con ello el proceso de descentralización.

Esta crisis estaría manifiesta en todos los sectores que si bien relevan lo normativo y los procedimientos administrativos institucionales, mencionan las necesidades de cambios en las estructuras y señalan los obstáculos al momento de intentar introducir modificaciones, las cuales se presentan en los distintos niveles siendo más percibidas las dificultades de la implementación de las políticas, quizás por el rol que corresponde a los decisores participantes.

En este sentido, se observarían dos tendencias contrapuestas. Por una parte, considerar los criterios institucionales sería funcional a los decisores, pues son estos los criterios con los que se evalúa su gestión, y/o siempre está la posibilidad de seguir un patrón establecido y no arriesgarse a innovaciones, que generalmente implican mayores costos y resistencias a los cambios. Por otra parte, sin embargo, se observa que las necesidades de cambios sugeridas por los participantes, se pudiesen relacionar con los costos que implica percibir las inconsistencias del sistema y en definitiva, trabajar en un sistema que se desea cambiar.

En esto es relevante observar la tendencia a responder en mayor medida a lo presente, lo coyuntural. Por ello, este estudio sugiere la necesidad evaluar el costo que implica escindir la decisión operativa concreta del futuro que se desea, es decir del para qué se esta decidiendo; lo operativo y la visión prospectiva que hay detrás.

Se considera, entonces, la necesidad de promover el reencantamiento con el servicio público sobre todo a partir de rescatar las visiones y los deseos de las personas encargadas y los decisores, de modo tal que las normativas y procedimientos institucionales se ajusten al o los proyectos de sociedad que se planteen y no al revés.

Como algunas iniciativas promotoras de estos cambios, podemos observar diferentes énfasis en los sectores. Así, el sector educación otorga importancia al trabajo interdisciplinario, observando la escasa existencia de diversidad de profesiones y por lo tanto miradas al interior de este sistema, lo que pudiera relacionarse con dificultades a la hora de intentar cambios. El sector salud releva el trabajo con la familia y en ello el visualizar los recursos de ésta y fortalecerla en su rol, mientras el sector gobierno plantea la relevancia del trabajo con la comunidad y la búsqueda del empoderamiento comunitario. En este 
sentido, se estima que los tres sistemas mencionados, señalan la necesidad de incorporar nueva información a su sistema, como una vía para favorecer los cambios.

Sin embargo, resulta complicado que cada sistema esté tan diferenciado, al momento de plantearse alternativas innovadoras, pues ello complejiza aún más la idea de un abordaje integral, haciéndose necesaria la presencia de puentes a fin de focalizar los intereses de los distintos subsistemas, en un mismo proyecto de desarrollo. En esto, se estima que un rol gravitante puede ser jugado por la territorialidad y la necesidad de fortalecer el desarrollo local.

Un aspecto que se repite tanto en el Delphi como en el taller y específicamente en relación al futuro deseado para los niños, niñas y adolescentes de la comuna, se refiere al cambio cultural y ello se podría relacionar con la necesidad de pensar el futuro relevando el rol de las personas. En este aspecto ya en el Delphi se plantean dentro de las estrategias favorecedoras de la implementación de la Convención el aumento de la socialización de la Convención y el paradigma de los derechos de las personas, y en ello la promoción de una nueva relación entre niños y adultos. En este sentido favorecer el desarrollo de las personas resulta ser tanto una necesidad de los adultos (incluidos los mismos decisores) como de los niños.

Lo anterior, sin embargo, no implica necesariamente la consideración del respeto por sus derechos civiles y políticos, aspecto que en un principio constituyó el objetivo de este estudio, pues su contribución a la comunidad desde su ser niño, niña y adolescente, no es percibida como tal en el presente (por lo cual no aparecieron datos suficientes al respecto), sino como la necesidad de invertir en la infancia y adolescencia para el futuro, observándose un mayor interés por la satisfacción de necesidades básicas y la superación de la pobreza, lo cual representa, sin duda una realidad contingente.

Sin embargo, apostando a un desarrollo sostenible no sólo en el mediano sino en el largo plazo, este estudio ha querido subrayar la importancia de los derechos civiles y políticos apreciando que dentro de los participantes de la investigación, se observan escasamente algunos contenidos relacionados con favorecer la participación en las comunidades de niños, niñas y adolescentes e incluso se menciona la escasa relevancia otorgada a centros de alumnos. Así mismo es necesario también enfatizar que ninguno de los grupos que trabajaron en el taller, consideró en la búsqueda de una solución a la problemática dada la participación del niño o adolescente involucrado. 
Los derechos civiles y políticos, no aparecen espontáneamente mencionados, no se observa a los niños, niñas y adolescentes como actores relevantes y se tiende a mantener una visión de niños víctima y/o una percepción de estos negativa, asociados a problemáticas sociales de delincuencia y drogas, entre otras. En este aspecto es relevante considerar la percepción de que estos niños se convertirían en padres desvalidos y que por tanto las pautas que obstaculizan los procesos de desarrollo pudiesen perpetuarse.

La inexistencia de la percepción de derechos civiles y políticos, en tanto, se estima estaría vinculado a creencias asociadas a la infancia y a la adolescencia, que no consideran su participación como sujetos activos y propositivos de su desarrollo, lo cual dificulta no sólo las capacidades para decidir considerando el «interés superior del niño", sino también para promover acciones y decisiones que apuesten a un futuro positivo planeado con y para los niños, niñas y adolescentes.

Del mismo modo, si bien en un inicio el estudio pretendió trabajar con los decisores de la comuna de Temuco, no fue posible acceder a decisores de la comuna solamente, manifestando los participantes que sus decisiones estaban supeditadas a las decisiones regionales o nacionales. Ello tendría directa relación con la lentitud del proceso descentralizador, observándose en los decisores una sensación de escasa toma de decisiones en la comuna respecto del tema, lo que implicaría un escaso empoderamiento al respecto y una menor apuesta en iniciativas de desarrollo local que pudiesen ser impulsadas por el ámbito local.

Lo anterior, cuestiona la capacidad que como sociedad tenemos de convertirnos en ciudadanos, partícipes activos de los procesos sociales, y formar desde esta perspectiva a nuestros miembros más jóvenes, fortaleciendo con ello la democracia y el desarrollo humano.

TEMUCO (CHILE), MARZO 2007

RECIBIDO: MARZO 2007

ACEPTADO: MAYO 2007 


\section{REFERENCIAS BIBLIOGRÁFICAS}

ALLAN, S. (1999): «Participación infanto-juvenil: un reto social». En: www.sistemas.ues.edu.sv.

GOBIERNO DE CHILE (2001): «Política nacional y plan de acción integrado a favor de la infancia y la adolescencia 2001-2010». Santiago: Ministerio de Planificación y Cooperación.

GODET, MiCHEL (1996): La caja de herramientas de la prospectiva estratégica. Zarautz: Instituto Europeo de Prospectiva y Estrategia.

IZUZQUIZA, IGNACIO (1990): La sociedad sin hombres, Niklas Luhmann o la teoría del escándalo. Barcelona: Antrophos.

PÉREZ-LuCO, R. (2002): Método de análisis de contenidos jerárquico ponderado. Temuco: Universidad de La Frontera.

RAMíREZ, S. (2002): Reforma del Estado y modernización de la gestión pública. Lecciones y aprendizaje de la experiencia chilena. Barcelona: Instituciones y Desarrollo.

SÁNCHEZ, A. (2002): «La política y programas sociales integrales de superación de la pobreza: un desafío a la gestión pública». En: www.ppu.cl.

SILVA, J. (2000): «Exclusión social y juventud popular». En: www.subversion.uchile.cl.

UNICEF (2002): Estado mundial de la infancia 2002: capacidad de liderazgo. En: www.unicef.org.

(2001): Nosotros los niños y las niñas. Cumplir las promesas de la Cumbre Mundial a favor de la Infancia. En: www.unicef.org. 